\title{
Ionic Conductivity and Morphological Study of a Thermoplastic Polyurethane Based Electrolyte Comprising of Mixed Soft Segments
}

\author{
Mohanlal DIGAR and Ten-Chin WEN ${ }^{\dagger}$ \\ Department of Chemical Engineering, \\ National Cheng Kung University, \\ Tainan 701, Taiwan
}

(Received March 6, 2000; Accepted July 20, 2000)

\begin{abstract}
The morphology and ionic conductivity of three thermoplastic polyurethane (TPU) based polymer electrolytes have been studied by complexing lithium per chlorate $\left(\mathrm{LiClO}_{4}\right)$ with these TPUs. The TPUs were prepared by using 4,4'-diphenyl methane diisocyanate (MDI) extended with ethylene diamine (EDA) as hard segment and polypropylene glycol (PPG), polytetramethylene glycol (PTMG) and 1:1 mixture of PPG/PTMG as soft segments. The characterization of these polymeric electrolytes doped with different concentrations of $\mathrm{LiClO}_{4}$ were studied by using differential scanning calorimetry (DSC), fourier transform infrared spectroscopy (FT-IR) and AC impedance measurements. Doping was done by two methods: (1) by blending TPU and $\mathrm{LiClO}_{4}$ solutions in dimethyl formamide (DMF), and (2) by immersing dry TPU films in $\mathrm{LiClO}_{4}$ solution in DMF. Ionic conductivity of all the polymeric electrolytes increases with increasing salt concentration for each isotherm and also with increase in amount of plasticizer. The polymeric electrolytes containing both PPG and PTMG as soft segment are found to have almost the same ionic conductivity as that containing only PPG in the soft segment. On the other hand, the dimensional stability of these electrolytes is comparable to that containing PTMG as the soft segment.

KEY WORDS Thermoplastic Polyurethane / Polymer Electrolyte / Ionic Conductivity / Dimensional Stability / Polypropylene Glycol / Polytetramethylene Glycol /
\end{abstract}

Ionically conducting polymers or polymer ionics, where one or more of alkali metal salts are dissolved in macromolecular solids, are known for many years. But interest on these materials was first initiated in 1951 when it was recognized ${ }^{1}$ that alkali metal ions interact with the ether oxygen of polyethylene oxide (PEO) chains. Few years later substantial research was undertaken by many researchers ${ }^{2-7}$ on the properties of these polymer salt solutions. However, the ionic conductivity of these materials was first reported by Wright et $a l^{8,9}$ in 1973. The initial realization that these materials could be used as electrolyte for battery applications was first reported by Armand et al. ${ }^{10,11} \mathrm{PEO}$, complexed with alkali metal salts remain to date the most widely studied polymer electrolyte from the theoretical and practical point of view. ${ }^{12,13}$ Because of the crystalline nature of $\mathrm{PEO}$, its room temperature conductivity is low $\left(<10^{-8} \mathrm{~S}\right.$ $\mathrm{cm}^{-1}$ ) and hence its use as polymer electrolyte in lithium batteries is restricted at higher temperature $(>80$ ${ }^{\circ} \mathrm{C}$ ) only. Recognizing that the ionic conductivity of polymer electrolytes is facilitated in the elastomeric amorphous phase by the segmental motion of the polymer chains, significant research effort has been devoted in tailoring polymer structure having highly flexible backbone and amorphous character. This has led to the discovery of poly[bis(methoxyethoxyethoxide)phosphazene] (MEEP), the Li-salt complexes of which are fully amorphous and exhibit a conductivity of $2 \times 10^{-5} \mathrm{~S} \mathrm{~cm}^{-1}$ at room temperature, which is one of the highest among the conventional polymer electrolytes. ${ }^{14}$

Although higher ionic conductivity is one of the most important prerequisites for a material to be used as polymer electrolyte, its dimensional stability is also equally important. Generally, ionic conductivity and dimensional stability of polymer electrolytes are inversely related, the higher the ambient temperature conductivity of the electrolyte, the lower is its dimensional stability. The ambient temperature conductivity of MEEP-Li salt complexes is among the highest reported to date. Conversely, their dimensional stability is among the poorest. It is not possible to use most of the MEEP-Li salt complexes as separators in batteries since the polymer and its $\mathrm{Li}$ salt complexes are glutinous materials having a tendency to flow even under mild pressure at ambient temperature. ${ }^{15}$

Linear segmented polyurethane is an important class of thermoplastic elastomers having general structure $(A-B)_{n}$. Here B is the soft segment usually formed from one or more of polyether or polyester polyol and $A$ is the hard segment formed by extending a diisocyanate with low molecular weight diol or diamine. In the segmented polyurethanes, phase separation of the urethane hard segments into micro domains has been observed ${ }^{16}$ even when the segment length is relatively short. The primary driving force for phase separation or domain formation is the strong intermolecular interactions of the urethane units, which are capable of forming interurethane hydrogen bonds. Factors that control the degree of micro phase separation include copolymer composition, segment length, crystallizability of either segment and the method of sample preparation. In general, interconnected or isolated hard segments remain distributed in the soft segment matrix, though the soft phase may contain some hard segments dissolved in it,

\footnotetext{
${ }^{\dagger}$ To whom correspondence should be addressed (Tel: 886-6-2757575, Extn. 62656, Fax: 886-6-2344496, E-mail: tcwen@mail. ncku.edu.tw).
} 
Table I. List of reagents and chemicals

\begin{tabular}{lll}
\multicolumn{1}{c}{ Designation } & \multicolumn{1}{c}{ Chemical identification } & \multicolumn{1}{c}{ Source } \\
\hline MDI & 4,4-diphenylmethane diisocyanate & DuPont Company, Inc. \\
PPG 2000 & Polypropylene glycol $\left(M_{\mathrm{w}}=2000\right)$ & DuPont Company, Inc. \\
PTMG 2000 & Polytetramethylene glycol & DuPont Company, Inc. \\
& $\left(M_{\mathrm{w}}=2000\right)$ & Aldrich Chemical Company, Inc. \\
$\mathrm{EDA}$ & Ethylene diamine & Mallinckredt Baker Inc. \\
$\mathrm{DMF}$ & Dimethylformamide & Mallinckredt Baker Inc. \\
$\mathrm{MeOH}$ & Methyl alcohol & Aldrich Chemical Company, Inc. \\
$\mathrm{LiClO}$ & Lithium perchlorate & Aldrich Chemical Company, Inc. \\
$\mathrm{PC}$ & Propylene Carbonate & \\
\hline
\end{tabular}

which is evident from the hydrogen bonding of the urethane-NH groups with the oxygen of the ether or ester linkages. ${ }^{17,18}$

Because of this unique two-phase microstructure, the segmented polyether urethanes find themselves very much useful as matrix material for polymer electrolyte. The rubbery soft segments can dissolve alkali metal salts without formation of ionic clusters, which may be due to the interaction of the ether oxygens with the alkali metal ions. ${ }^{3,19}$ Furthermore, the low glass transition temperature $\left(T_{\mathrm{g}}\right)$ and hence higher segmental motion of these polyethers lead to higher mobility of the dissolved ions. The ability to dissolve alkali metal ions along with higher ionic mobility results in a relatively high ionic conductivity, which is one of the most important prerequisites for the polymer salt complexes to be used as polymer electrolyte. The hard segment domains, which are in the glassy state and are either distributed or interconnected throughout the rubbery phase of the soft segment, act as reinforcing filler and hence contribute to the dimensional stability of the polymer electrolytes.

Our research presents the efficacy of a 1:1 polypropylene glycol (PPG) and polytetramethylene glycol (PTMG) based thermoplastic polyurethane (TPU) as matrix material for polymer electrolyte over the two parent TPUs containing either PPG or PTMG. The TPUs made for this purpose are composed of $4,4^{\prime}$-diphenyl methane diisocyanate (MDI) extended with ethylene diamine (EDA) as hard segment and either PPG or PTMG or a 1:1 mixture of both as soft segment. The hard to soft segment molar ratio was always maintained at $3: 1$. The morphology and ionic conductivity of the undoped and $\mathrm{LiClO}_{4}$ doped TPUs were investigated by differential scanning calorimetry (DSC), fourier transform infrared spectroscopy(FT-IR) and AC impedance measurements. The effect of doping method on the conductivity of these solid polymer electrolytes at their various doping levels has also been studied. The three TPUs made by using PTMG, PPG and 1:1 mixture of PTMG and PPG are designated as TPU 1, TPU 2, and TPU 3, respectively.

\section{EXPERIMENTAL}

The chemicals and reagents used in this study are given in Table I. PPG and PTMG were dehydrated under reduced pressure at $80^{\circ} \mathrm{C}$ for $24 \mathrm{~h}$ before use. $\mathrm{LiClO}_{4}$ was dehydrated at $120^{\circ} \mathrm{C}$ under reduced pressure for 72 h. All other reagents and solvents were used without further purification.

The TPUs were synthesized by a two-step addition process, where the prepolymer was made in the first step by reaction of excess of MDI with the polyols and then extending the chain length by reaction of the prepolymer with the chain extender in the second step. The prepolymers were made by allowing the mixture of MDI and polyol/s to react at $85^{\circ} \mathrm{C}$ for $6 \mathrm{~h}$ with stirring under a constant dry nitrogen blanket. After the prepolymer formation was over, it was dissolved in DMF and the temperature of the mass was reduced to $-5^{\circ} \mathrm{C}$. Then a 3 wt\% solution of EDA in DMF was slowly added to the prepolymer solution at $-5^{\circ} \mathrm{C}$ with constant stirring. After the addition of EDA solution was over, the temperature of the mass was increased to $85^{\circ} \mathrm{C}$ and the reaction was allowed to continue for $1 \mathrm{~h}$ at this temperature. The concentration of the polymer at this stage was $\sim 20 \mathrm{wt} \%$. The polymer solution was cooled to room temperature and precipitated into excess methanol. It was reprecipitated twice from dimethyl formamide (DMF) into excess methanol, washed several times with methanol and dried in vacuum at $100^{\circ} \mathrm{C}$ for $72 \mathrm{~h}$.

Characteristics of the TPUs made are summarized in Scheme 1 and Table II. Molar ratio of MDI, polyol and the chain extender was always maintained at 3:1:2. In TPU 3, PPG and PTMG were used in 1:1 molar ratio. The molecular weights and the molecular weight distributions of the TPUs were measured using a Shimadzu Gel Permeation Chromatograph (GPC) connected to a Shimadzu high performance liquid chromatography (HPLC) pump and an RI detector. $1 \mathrm{wt} \%$ solution of the TPUs in DMF were injected into the GPC column (Jordi Gel DVB mixed bed) at a flow rate of $2 \mathrm{~mL} \mathrm{~min}^{-1}$. The system was calibrated with polystyrene standards (Aldrich).

Films for DSC and AC impedance measurements were made on glass substrates by casting from a $10 \mathrm{wt} \%$ solution of the TPUs in DMF. For doping by solution blending, required amount of $10 \mathrm{wt} \%$ solution of $\mathrm{LiClO}_{4}$ in DMF was blended with the TPU solutions by stirring for $12 \mathrm{~h}$ with the help of magnetic stirrer. For doping by absorption method, the preweighed undoped dry films were dipped in 10 wt\% $\mathrm{LiClO}_{4}$ solution in DMF, excess solution was soaked with filter paper and then reweighed. Drying of the doped as well as the undoped films was done at $100^{\circ} \mathrm{C}$ under vacuum for $72 \mathrm{~h}$. The gelled polymer electrolytes were prepared from solid polymer electrolytes by dripping required amount of propylene carbonate (PC) on the doped films and by allowing it to stand up to $2 \mathrm{~h}$ for complete absorption of $\mathrm{PC}$. For FT-IR analysis, films were cast on $\mathrm{KBr}$ pellets from a $5 \mathrm{wt} \%$ solution in DMF and dried at $120^{\circ} \mathrm{C}$ under vac- 

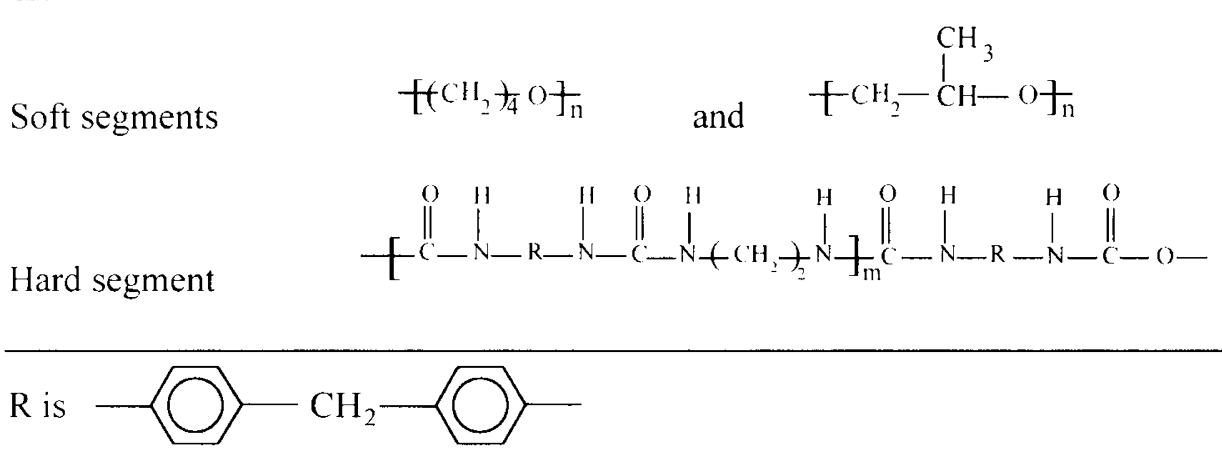

Scheme 1. Schemetic representation of soft and hard segments.

Table II. Characterization of the TPUs

\begin{tabular}{lccrc}
\hline TPU & $\begin{array}{c}\text { Molar ratio } \\
\text { (MDI:polyol:EDA) }\end{array}$ & $M_{\mathrm{n}}$ & \multicolumn{1}{c}{$M_{\mathrm{w}}$} & PDI $^{\mathrm{a}}$ \\
\hline TPU 1 & $3: 1: 2$ & 28775 & 54600 & 1.90 \\
TPU 2 & $3: 1: 2$ & 62661 & 113305 & 1.81 \\
TPU 3 & $3: 1: 2$ & 74359 & 141851 & 1.91 \\
\hline
\end{tabular}

${ }^{\mathrm{a}}$ Polydispersity index.

uum for $72 \mathrm{~h}$. The samples were then shifted to the glove box where it were transferred into air tight containers to avoid absorption of moisture. The containers were opened only at the time of measurement of FT-IR spectra.

FT-IR spectra were measured at ambient temperature using a Nicolet 550 system with a wave number resolution of $4 \mathrm{~cm}^{-1} .132$ scans were signal averaged to increase the signal to noise ratio. The $-\mathrm{NH}$ and $>\mathrm{C}=\mathrm{O}$ stretching regions were deconvoluted using a commercial software (Grams 386 software from Galactic Industries Corporation). The maximum error associated with the deconvolution studies is expected to be $\pm 10 \%$.

DSC measurements were made using a DSC 2010 Differential Scanning Calorimeter (TA Instruments, USA) over a temperature range from -100 to $100^{\circ} \mathrm{C}$ at a scan rate $10^{\circ} \mathrm{C}$ per minute. All thermograms were base line corrected and calibrated using Indium metal. Experimental specimens ( 3 to $5 \mathrm{mg}$ ) were cut from cast films dried at $100^{\circ} \mathrm{C}$ under vacuum for $72 \mathrm{~h}$. Glass transition temperature was reported for the midpoint of the transition process, and the melting temperature was the peak temperature. As $T_{\mathrm{g}}$ of the soft segments of TPUs is reported ${ }^{27}$ to increase on annealing at higher temperature due to temperature induced phase mixing of the hard and soft segments, DSC measurements were made without annealing.

Impedance measurements of the solid as well as the gelled polymer electrolytes made from the TPUs were performed using thin films. Film thickness was maintained in the range of 150 to $200 \mu$ and the area of contact was $0.785 \mathrm{sq} \mathrm{cm}$. Samples were sandwiched between two stainless steel electrodes and fixed in an airtight double wall glass cell, through the outer jacket of which thermostated water was circulated for measurements at different temperatures. Cells were assembled in dry argon atmosphere inside a glove box (Vacuum Atmosphere Company, USA). Ionic conductivity of the samples was measured using an Autolab PGSTAT 30 equipment (Eco
Chemie B. V., Netherlands) with the help of Frequency Response Analysis system software under an oscillation potential of $250 \mathrm{mV}$.

\section{RESULTS}

\section{$F T-I R$}

The effect of salt concentration and doping method on the phase behavior of the TPUs has been investigated by FT-IR spectroscopy. In order to gain insight into the effect of $\mathrm{Li}^{+}$ions on the $\mathrm{H}$-bonding interaction of the hard and soft segments, two regions of the FT-IR spectra have been chosen: (1) $3700-3200 \mathrm{~cm}^{-1}$, the region for free and H-bonded - $\mathrm{NH}$ vibrations; and (2) 1750-1600 $\mathrm{cm}^{-1}$, the region for urethane and urea carbonyl symmetric stretching vibrations. Figure 1 shows the IR spectra of the $-\mathrm{NH}$ stretching region of TPU 3 in its doped and undoped form. Other samples are also having similar FT-IR spectra and hence not shown. The deconvolution results of all the samples are given in Table III. Deconvolution of carbonyl region of the FT-IR spectra has been done in two different regions: the urethane carbonyl region $\left(1760-1700 \mathrm{~cm}^{-1}\right)$, and the urea carbonyl region $\left(1700-1620 \mathrm{~cm}^{-1}\right)$. The deconvoluted spectra of TPU 3 (both undoped and doped) in the urethane carbonyl region is presented in Figure 2. It is evident from Figure 2 that the urethane carbonyl region is composed of three characteristic peaks. The three peaks in the regions $1737-1732 \mathrm{~cm}^{-1}, 1729-1722 \mathrm{~cm}^{-1}$, and $1709-$ $1705 \mathrm{~cm}^{-1}$ are assigned to free urethane carbonyl, disordered $\mathrm{H}$-bonded carbonyl and ordered $\mathrm{H}$-bonded carbonyl stretching vibrations respectively. ${ }^{33}$ To avoid overcrowding, the spectra for the urea carbonyl region of TPU 3 only, in its undoped and doped form, are presented in Figure 3. The results of deconvolution of the urethane and urea carbonyl absorptions for all the samples are presented in Table IV and V, respectively.

\section{$D S C$}

The thermal behavior of the TPUs has been studied by DSC and the results are presented in Table VI. Multiple transitions associated to both hard and soft segments of TPU have been reported in the literature. ${ }^{23-28}$ In this study, the thermal behavior of the soft segment only has been taken into consideration. TPU 2, containing fully amorphous PPG as the soft segment, shows only one endothermic transition corresponding to the $T_{\mathrm{g}}$ of PPG 2000. TPU 1 and TPU 3 show another endothermic tran- 


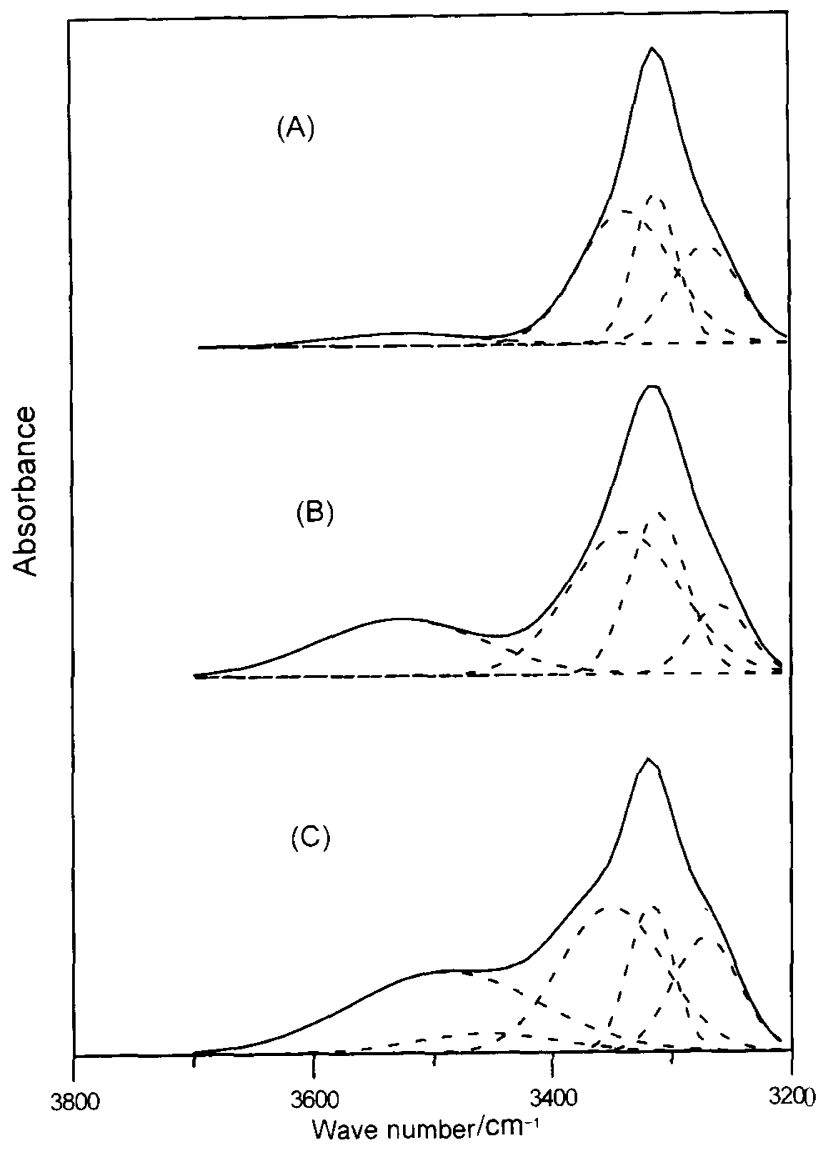

Figure 1. Deconvolution of $-\mathrm{NH}$ stretching region of TPU 3 (A)

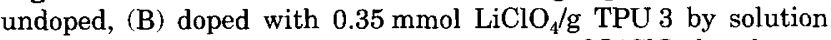
blending, and (C) doped with same amount of $\mathrm{LiClO}_{4}$ by absorption.

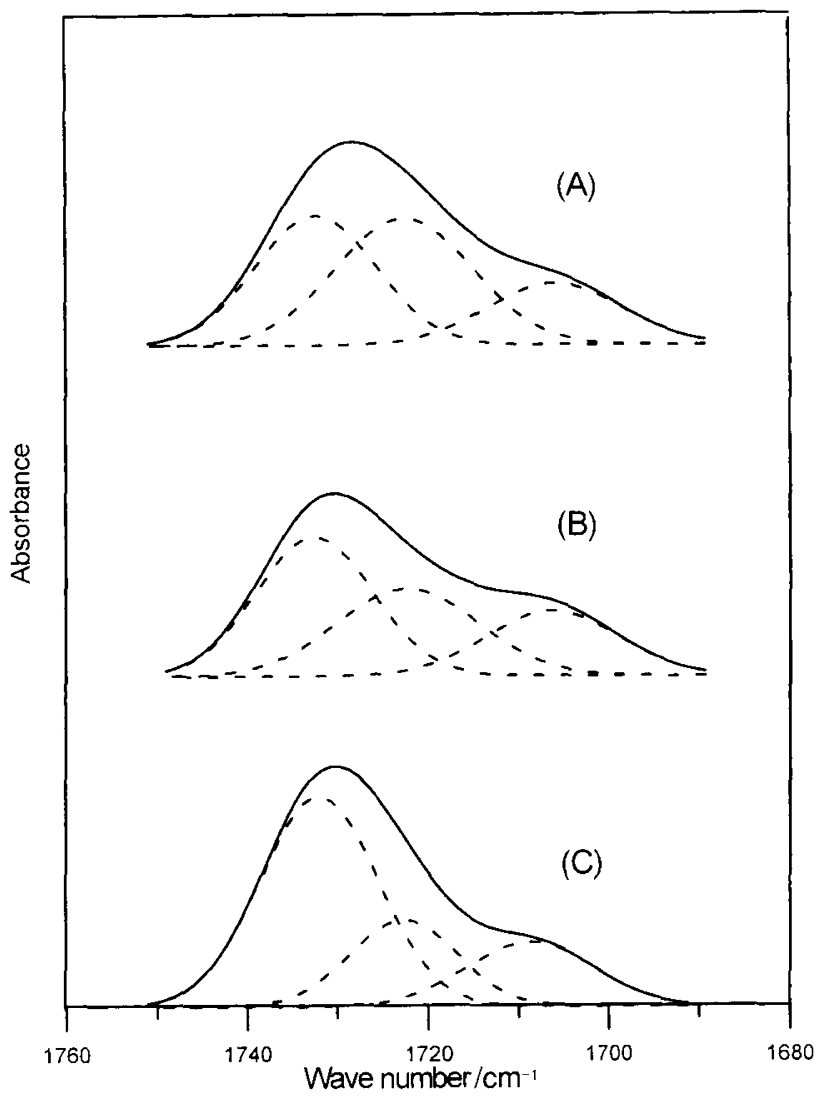

Figure 2. Deconvolution of $>\mathrm{C}=\mathrm{O}$ stretching region of TPU 3 (A) undoped, (B) doped with $0.35 \mathrm{mmol} \mathrm{LiClO}_{4} / \mathrm{g}$ TPU 3 by solution blending, and $(\mathrm{C})$ doped with same amount of $\mathrm{LiClO}_{4}$ by absorption.

Table III. Deconvolution results of the FT-IR spectra in the $-\mathrm{NH}$ stretching region

\begin{tabular}{|c|c|c|c|c|c|}
\hline \multirow{2}{*}{ Sample } & \multirow{2}{*}{$\begin{array}{l}\text { Salt conc./ } \\
\mathrm{mmol} \mathrm{g}^{-1}\end{array}$} & \multicolumn{4}{|c|}{ Peak position $/ \mathrm{cm}^{-1}$} \\
\hline & & 1 & 2 & 3 & 4 \\
\hline TPU 1 & 0.00 & 3528.2 & 3340.4 & 3316.3 & 3267.9 \\
\hline TPU 1-S1 & 0.15 & 3468.0 & 3355.7 & 3318.9 & 3273.5 \\
\hline TPU 1-S2 & 0.35 & 3462.5 & 3391.4 & 3313.0 & 3265.6 \\
\hline TPU 1-A1 & 0.15 & 3488.8 & 3353.8 & 3319.3 & 3272.7 \\
\hline TPU 1-A2 & 0.36 & 3486.1 & 3352.2 & 3320.1 & 3274.7 \\
\hline TPU 2 & 0.00 & 3527.4 & 3343.5 & 3316.3 & 3269.0 \\
\hline TPU 2-S1 & 0.15 & 3522.2 & 3347.8 & 3310.9 & 3259.0 \\
\hline TPU 2-S2 & 0.35 & 3512.5 & 3354.1 & 3311.6 & 3260.9 \\
\hline TPU 2-A1 & 0.15 & 3528.3 & 3343.9 & 3312.9 & 3263.8 \\
\hline TPU 2-A2 & 0.35 & 3527.7 & 3341.2 & 3312.4 & 3261.0 \\
\hline TPU 3 & 0.00 & 3529.5 & 3346.0 & 3318.8 & 3277.6 \\
\hline TPU 3-S1 & 0.15 & 3507.8 & 3346.4 & 3318.0 & 3275.1 \\
\hline TPU 3-S2 & 0.35 & 3527.8 & 3339.4 & 3312.4 & 3260.9 \\
\hline TPU 3-A1 & 0.16 & 3499.8 & 3344.1 & 3318.1 & 3275.1 \\
\hline TPU 3-A2 & 0.35 & 3487.9 & 3354.6 & 3317.6 & 3271.7 \\
\hline
\end{tabular}

A1, A2: samples doped by absorption method; S1, S2: samples doped by solution blending.

sition apart from the $T_{\mathrm{g}}$ of the soft segment. Due to the presence of semi crystalline PTMG 2000, one crystalline melting transition was observed for these two TPUs. The data presented in Table VI indicates an increase in soft segment $T_{\mathrm{g}}$ with the increase in salt concentration. Moreover, the increase in $T_{\mathrm{g}}$ with salt concentration is more when doped by solution blending compared to that by absorption method.

\section{AC Impedance}

AC conductivity of TPU 1 , TPU 2 , and TPU 3 , as a function of reciprocal temperature, has been presented 

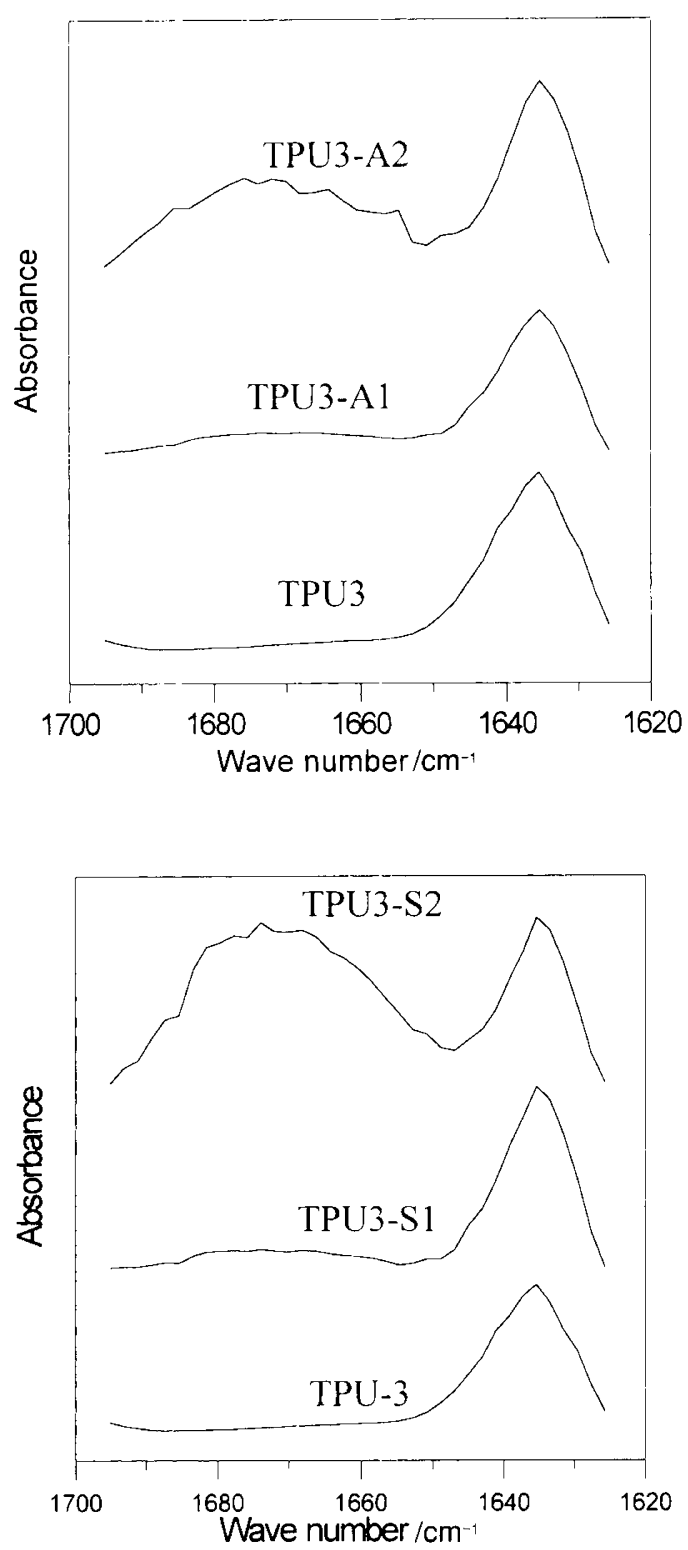

Figure 3. FT-IR spectra of TPU 3 in the urea $>C=0$ stretching region: (A) doped by absorption and (B) doped by solution blending.

in Figures 4, 5, and 6 respectively. Conductivity of each of the doped samples has been found to increase linearly with the increase in temperature. However, there is one exception (Figure 5A), where the temperature dependence is not linear. The temperature dependence of ionic conductivity of all the samples in Figures $4-6$ follow the Arrhenius eq 1, whereas the samples presented in Figure 5A follow the Vogel-Tamman-Fulcher (VTF) eq 2.

$$
\begin{aligned}
& \sigma(T)=A \exp \left[-E / k_{\mathrm{B}} T\right] \\
& \sigma(T)=A T^{-1 / 2} \exp \left[-B / k_{\mathrm{B}}\left(T-T_{0}\right)\right]
\end{aligned}
$$

where $\sigma(T)$ is the conductivity at temperature $T, A$ is a constant proportional to the number of charge carriers, $E$ is the activation energy, $k_{\mathrm{B}}$ is the Boltzmann constant, $B$ is the pseudo activation energy related to polymer segmental motion and $T_{0}$ is the temperature at which the configurational entropy of the polymer becomes zero and is close to the glass transition temperature.

Figure 7 presents the comparative conductivity data for the three TPUs having almost identical concentration of Li-salt, doped by both absorption and solution blending method. It is evident from Figure 7 that for both type of doping processes, conductivity varies in the order TPU $2>$ TPU $3>$ TPU 1 for a particular doping level. Moreover, for a particular concentration of Li-salt, conductivity was found to be higher when doped by solution blending compared to that by absorption method.

As the ionic conductivities of the solid polymer electrolytes (SPE) are very low $\left(\sim 10^{-6} \mathrm{~S} \mathrm{~cm}^{-1}\right.$ at $\left.85^{\circ} \mathrm{C}\right)$, attempt has been made to see the efficacy of these systems as gelled polymer electrolytes (GPE). As the TPU 3 system possesses the combination of high conductivity of TPU 2 and good dimensional stability of TPU 1 , only TPU 3 has been tested as GPE. Figures 8 and 9 show the temperature dependence of ionic conductivity of TPU 3S2 and TPU 3-A2, respectively, containing different amount of added propylene carbonate (PC) as plasticizer. As is evident from Figures 8 and 9, the GPEs having lower concentration of PC show VTF like temperature dependence, whereas at higher concentration, transformation into Arrhenius like behavior has been observed.

\section{DISCUSSION}

\section{$F T-I R$}

-NH Stretching Region. As is evident from Figure 1 and Table III, deconvolution of the FT-IR spectra of the TPUs in the $-\mathrm{NH}$ stretching region results four characteristic peaks. Out of these four peaks, peak 1 in the region of $3530-3525 \mathrm{~cm}^{-1}$, which appears as a low intensity shoulder for all the three undoped TPUs, is assigned to free $-\mathrm{NH}$ stretching vibration. The peak position for the $\mathrm{H}$-bonded stretching vibration has been reported ${ }^{20,21}$ to be in the region of $3320-3310 \mathrm{~cm}^{-1}$. Colemann et $a l^{21}$ reported, from the study of their model system (Ethyl urethane-Tetrahydrofuran), that the $-\mathrm{NH}$ to ether oxygen interaction is comparable to that of $-\mathrm{NH}$ to carbonyl oxygen interaction. Hence, the $-\mathrm{NH}$ groups $\mathrm{H}$ bonded to either ether or carbonyl oxygens should give same absorption peak. But Lee et $a l^{22}$ and later Wen et $a l .{ }^{35}$ reported the existence of another absorption peak in the region of $3295-3260 \mathrm{~cm}^{-1}$, which has been assigned for the $-\mathrm{NH}$ stretching vibration where the $-\mathrm{NH}$ groups are H-bonded to the ether oxygen atoms. The peaks centered around $3320-3310 \mathrm{~cm}^{-1}$ (peak 3) and 3278-3260 $\mathrm{cm}^{-1}$ (peak 4) for our TPUs are thus assigned to the -NH stretching vibrations where the H-bondings are formed with the carbonyl and ether oxygens, respectively. Peak 2 , in the region of $3380-3340 \mathrm{~cm}^{-1}$ can be assigned as the overtone to the fundamental in the carbonyl region. ${ }^{20}$

Due to the similar chemical structure of the TPUs, all the $-\mathrm{NH}$ absorption bands are almost in the same position in the undoped state. It means that the strength of the free as well as $\mathrm{H}$-bonded $\mathrm{N}-\mathrm{H}$ band is not affected by the change of the soft segment. TPU 1, which contains PTMG as the soft segment, shows shifting of the free $-\mathrm{NH}$ band position to lower frequency with increase in salt concentration. The shifting is more pronounced 
Table IV. Deconvolution results of the FT-IR spectra in the $>\mathrm{C}=\mathrm{O}$ stretching region

\begin{tabular}{lcccc}
\hline Sample & $\begin{array}{c}\text { Salt conc. } \\
\text { mmol g }^{-1}\end{array}$ & \multicolumn{1}{c}{ Peak position / $\mathrm{cm}^{-1}$} & 2 \\
\hline TPU 1 & 0.00 & 1737.6 & 1729.4 & 1709.6 \\
TPU 1-S 1 & 0.15 & 1733.6 & 1727.7 & 1709.4 \\
TPU 1-S 2 & 0.35 & 1734.8 & 1727.2 & 1708.8 \\
TPU 1-A 1 & 0.15 & 1737.7 & 1730.1 & 1709.3 \\
TPU 1-A 2 & 0.35 & 1737.0 & 1729.8 & 1707.4 \\
TPU 2 & & & 1723.5 & 1705.8 \\
TPU 2-S 1 & 0.00 & 1733.5 & 1724.7 & 1707.1 \\
TPU 2-S 2 & 0.15 & 1733.8 & 1724.4 & 1706.0 \\
TPU 2-A 1 & 0.35 & 1733.4 & 1723.8 & 1706.1 \\
TPU 2-A 2 & 0.15 & 1733.0 & 1722.4 & \\
TPU 3 & 0.36 & 1732.3 & & 1719.7 \\
TPU 3-S 1 & & & 1721.8 & 1705.4 \\
TPU 3-S 2 & 0.00 & 1732.0 & 1722.4 & 1706.9 \\
TPU 3-A 1 & 0.15 & 1732.0 & 1723.2 & 1708.3 \\
TPU 3-A 2 & 0.35 & 1732.2 & 1724.0 & 1708.9 \\
\hline
\end{tabular}

A1, A2: samples doped by absorption method; S1, S2: samples doped by solution blending.

Table V. Deconvolution results of the FT-IR spectra in the urea $>\mathrm{C}=\mathrm{O}$ stretching region

\begin{tabular}{|c|c|c|c|c|c|}
\hline & TPU 1 & TPU 1-A1 & TPU 1-A2 & TPU 1-S1 & TPU 1-S2 \\
\hline & \multicolumn{5}{|c|}{ Peak positions } \\
\hline Peak 1 & 1636.7 & 1637.0 & 1636.9 & 1636.0 & 1636.4 \\
\hline \multirow[t]{2}{*}{ Peak 2} & - & 1664.7 & 1664.8 & 1668.4 & 1665.2 \\
\hline & \multicolumn{5}{|c|}{ Percent area } \\
\hline Peak 1 & 100.00 & 28.05 & 26.88 & 24.27 & 15.49 \\
\hline \multirow[t]{3}{*}{ Peak 2} & - & 72.95 & 73.12 & 75.72 & 84.51 \\
\hline & TPU 2 & TPU 2-A1 & TPU 2-A2 & TPU 2-S1 & TPU 2-S2 \\
\hline & \multicolumn{5}{|c|}{ Peak positions } \\
\hline Peak 1 & 1638.1 & 1634.0 & 1633.7 & 1633.7 & 1633.3 \\
\hline \multirow[t]{2}{*}{ Peak 2} & - & - & - & - & 1677.2 \\
\hline & \multicolumn{5}{|c|}{ Percent area } \\
\hline Peak 1 & 100.00 & 100.00 & 100.00 & 100.00 & 75.42 \\
\hline \multirow[t]{3}{*}{ Peak 2} & - & - & - & - & 24.58 \\
\hline & TPU 3 & TPU 3-A1 & TPU 3-A2 & TPU 3-S1 & TPU 3-S2 \\
\hline & \multicolumn{5}{|c|}{ Peak positions } \\
\hline Peak 1 & 1635.6 & 1635.4 & 1635.1 & 1635.9 & 1635.3 \\
\hline \multirow[t]{2}{*}{ Peak 2} & - & 1670.8 & 1670.9 & 1665.4 & 1671.3 \\
\hline & \multicolumn{5}{|c|}{ Percent area } \\
\hline Peak 1 & 100.00 & 66.66 & 41.76 & 79.81 & 25.96 \\
\hline Peak 2 & - & 33.34 & 58.24 & 20.19 & 74.04 \\
\hline
\end{tabular}

when doping was done by solution blending method. This may be attributed to the coordination of $\mathrm{Li}^{+}$ions to the nitrogen of the free $-\mathrm{NH}$ groups (Scheme 2a). Due to this ionic coordination to the $\mathrm{N}$-atoms, the $\mathrm{N}-\mathrm{H}$ bonds become weaker and hence the band position is shifted to lower frequency. ${ }^{33}$ Peak 4 shows slight shifting when doped by solution blending and when the salt concentration is relatively higher. Under such conditions, the $\mathrm{Li}^{+}$ ions may interact with the otherwise difficultly accessible soft segment ether oxygens due to the more rigid nature of PTMG. Shifting of the band position to lower frequency indicates that the $\mathrm{N}-\mathrm{H}$ bond $\mathrm{H}$-bonded to the ether oxygens is weakened due to the inductive effect of coordination of $\mathrm{Li}^{+}$ions with the electron rich oxygen atoms of the ether groups ${ }^{33}$ (Scheme $2 b$ ).
In contrast to TPU 1 , TPU 2 shows shifting of $\mathrm{H}$ bonded $-\mathrm{NH}$ peak positions with increasing salt concentration, whereas the free $-\mathrm{NH}$ peak position remained almost unaltered. This may be attributed to the fact that TPU 2 is softer in nature and hence all the coordinating sites are equally accessible to the $\mathrm{Li}^{+}$ions. Thus, the $\mathrm{Li}^{+}$ions may get the opportunity to coordinate preferentially to the more electronegative oxygen atoms and not the nitrogen atoms. TPU 3, containing both PPG and PTMG in the soft segment shows intermediate behavior of TPU 1 and TPU 2. The peak position for free $-\mathrm{NH}$ is shifted to lower frequency with addition of $\mathrm{LiClO}_{4}$ (like TPU 1). Unlike TPU 1 (but like TPU 2), both the Hbonded peaks are shifted to lower frequency, at least when salt concentration was relatively high and doping 
Table VI. Thermal transition temperatures of $\mathrm{LiClO}_{4}$ doped TPUs

\begin{tabular}{|c|c|c|c|c|}
\hline Sample & $\begin{array}{l}\text { Salt conc.' } / \\
\mathrm{mmol} \mathrm{g}^{-1}\end{array}$ & $T_{\mathrm{g}}{ }^{\circ} \mathrm{C}$ & $\Delta T_{\mathrm{g}}{ }^{\circ} \mathrm{C}$ & $T_{\mathrm{m}} /{ }^{\circ} \mathrm{C}$ \\
\hline TPU 1 & 0.00 & -77.54 & - & 7.24 \\
\hline TPU 1-S1 & 0.15 & -74.61 & 2.93 & 11.25 \\
\hline TPU 1-S2 & 0.35 & -74.39 & 0.22 & 13.25 \\
\hline TPU 1-A1 & 0.15 & -74.76 & 2.78 & 11.04 \\
\hline TPU 1-A2 & 0.35 & -75.33 & - & 4.56 \\
\hline TPU 2 & 0.00 & -52.91 & - & - \\
\hline TPU 2-S1 & 0.15 & -51.34 & 1.57 & - \\
\hline TPU 2-S2 & 0.35 & -48.21 & 3.13 & - \\
\hline TPU 2-A1 & 0.15 & -52.00 & 0.91 & - \\
\hline TPU 2-A2 & 0.36 & -50.55 & 2.36 & 一 \\
\hline TPU 3 & 0.00 & -64.91 & - & 12.68 \\
\hline TPU 3-S1 & 0.15 & -64.53 & 0.38 & 11.52 \\
\hline TPU 3-S2 & 0.35 & -63.17 & 1.36 & 12.55 \\
\hline TPU 3-A1 & 0.16 & -62.44 & 2.47 & 11.47 \\
\hline TPU 3-A2 & 0.35 & -61.43 & 1.01 & 13.65 \\
\hline
\end{tabular}

A1, A2: samples doped by absorption method; $\mathrm{S} 1, \mathrm{~S} 2$ : samples doped by solution blending.

was done by solution blending method. These results reveal that TPU 3-S2 with both PPG and PTMG in the soft segment gives a more favorable environment for $\mathrm{Li}^{+}$ ions to interact with all the coordinating sites (Scheme 1) and hence gives rise to higher conductivity with desirable dimensional stability, which can also be explained from our DSC and AC impedance data.

$>C=O$ Stretching Region. The urethane carbonyl absorption region (Figure 2 and Table IV) shows no change in position of any of the sub peaks with salt concentration. However, the ordered $\mathrm{H}$-bonded urethane carbonyl peak for TPU 1-S2 is not detected. The urea carbonyl absorption region of the undoped TPUs shows only one peak in $1638-1636 \mathrm{~cm}^{-1}$ region, which may be due to H-bonded urea carbonyl absorption. ${ }^{37}$ With addition of salt, another peak arises in $1671-1664 \mathrm{~cm}^{-1}$ region, which may be due to the absorption of free urea carbonyl groups. From the peak area data (Table V) it is evident that the intensity of absorption of the free urea carbonyl peak is gradually increased with salt concentration and the same for the H-bonded urea carbonyl is decreased with increase in salt concentration. This may be attributed to some loss of $\mathrm{H}$-bonded species due to coordination by $\mathrm{Li}^{+}$ions with the $\mathrm{H}$-bonded urea carbonyls. It is also to be noted that the interaction of $\mathrm{Li}^{+}$ions with the urea carbonyls is in the order TPU $1>$ TPU $3>$ TPU 2. In case of TPU 2, no absorption for free urea carbonyl has been observed even in presence of $\mathrm{Li}$-salt, except in TPU 2-S2, where the salt concentration was relatively high and doping was done by solution blending method. For TPU 2, having PPG in the soft segment, most of the $\mathrm{Li}^{+}$ions may coordinate with the soft segment ether groups leaving the $\mathrm{H}$-bonded urea groups undisturbed. Hence no absorption was observed for free urea carbonyls.

\section{$D S C$}

As is evident from Table VI, the soft segment $T_{\mathrm{g}}$ is increased with increase in salt concentration and the effect is more when doping was done by solution blending method. Increase in $T_{\mathrm{g}}$ of the soft segment of TPU based electrolytes is reported earlier by many researchers ${ }^{29-33}$ which has been described as due to coordination of ether oxygens by $\mathrm{Li}^{+}$ions. Coordination by $\mathrm{Li}^{+}$ ions partially arrests the segmental motion of the soft segments, resulting in an increase in the $T_{\mathrm{g}}$. As the concentration of salt is increased, more and more cross links are formed and hence the $T_{\mathrm{g}}$ is increased with increase in salt concentration. An examination of the $\Delta T_{\mathrm{g}}$ values (Table VI) indicates that doping by solution blending leads to increased effect on $T_{\mathrm{g}}$ compared to that by absorption method. These results are very much in line with the general concept that interaction between two species is more pronounced in solution phase rather than in a solid-liquid interface. When the salt solution is blended with TPU solution, the $\mathrm{Li}^{+}$ions get easier access to the soft segment interacting sites because of the higher segmental motion of the TPU chains in solution phase. But when the TPU dry films are immersed in the salt solution, the $\mathrm{Li}^{+}$ions' accessibility to the soft segment interacting sites is a bit restricted because of the restricted segmental motion of the TPU chains in solid dry films.

The variation of $T_{\mathrm{g}}$ values for TPU 3 with salt concentration is a bit anomalous for two different methods of doping. Contrary to the behavior of TPU 1 and TPU 2, doping by absorption leads to more increase in $T_{\mathrm{g}}$ values than by solution blending. A possible explanation may be, due to the crystalline nature of the soft segment of TPU 3 (as is evident from the crystalline melting temperatures in the region of 10 to $15^{\circ} \mathrm{C}$ ), the $\mathrm{Li}^{+}$ions are preferentially coordinated to the PPG ether oxygens when doped by means of absorption and hence the $T_{\mathrm{g}}$ increase is more in this case compared to those in case of solution blending. When solution blended, both the PPG and PTMG ether oxygens are almost equally available to $\mathrm{Li}^{+}$ions. There might be some competition of the PPG and PTMG ether oxygens to be coordinated with the $\mathrm{Li}^{+}$ ions and hence the resultant increase in $T_{\mathrm{g}}$ is a bit lower than that in case of doping by absorption method. 

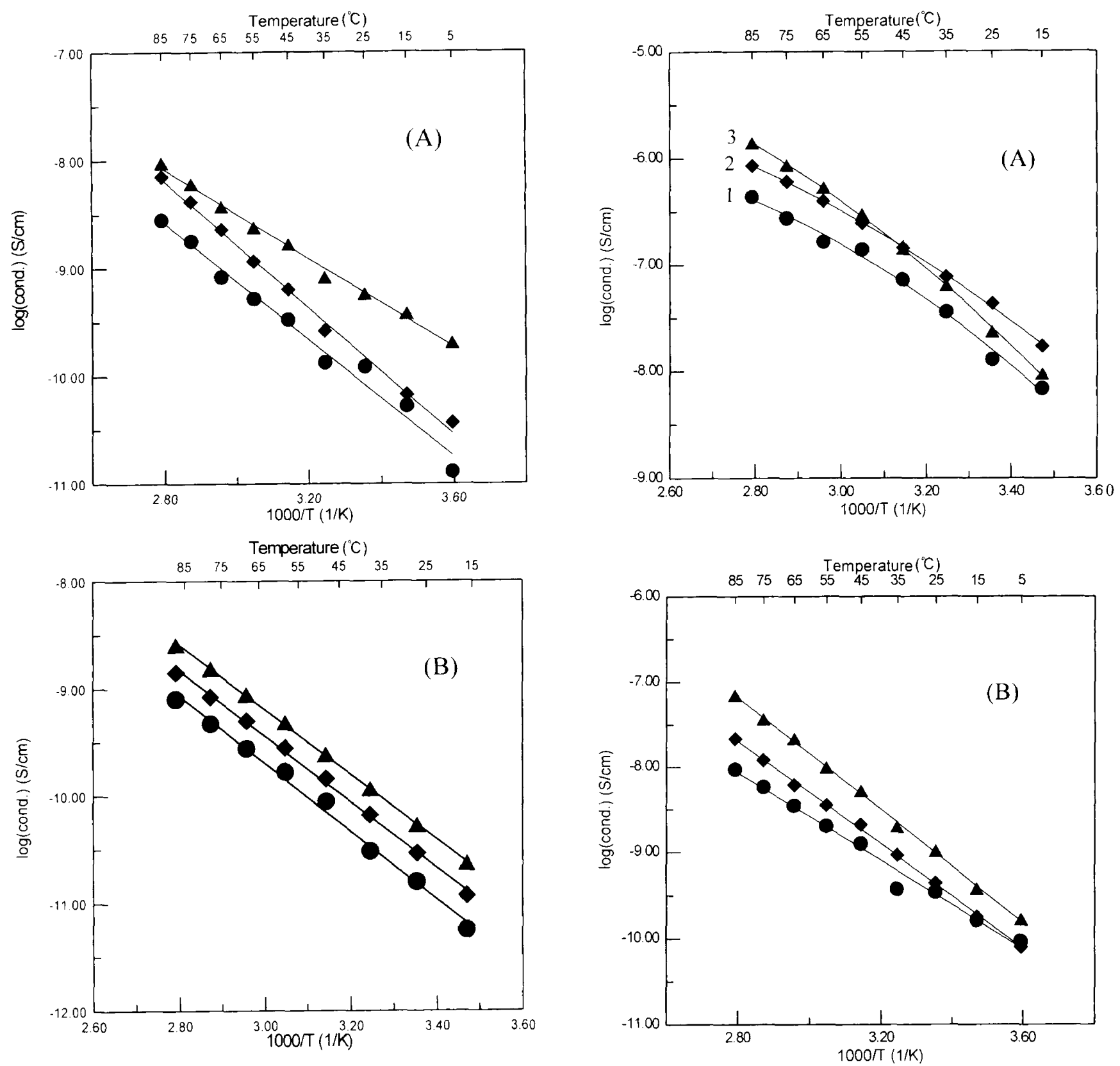

Figure 4. Temperature dependence of conductivity of TPU 1 with various concentration of Li-perchlorate, $0.15 \mathrm{mmol} \mathrm{g}^{-1}$, $0.25 \mathrm{mmol} \mathrm{g}^{-1}$, and $\Delta 0.35 \mathrm{mmol} \mathrm{g}^{-1}$. (A) doped by solution blending and (B) doped by absorption.

\section{AC Impedance}

Ionic conductivity of TPU based electrolytes as a function of temperature has been reported by many researchers. ${ }^{29-35}$ The linear dependence of ionic conductivity on temperature of most of our TPU electrolytes is consistent with the thermally activated behavior generally observed for an amorphous polymer above its glass transition temperature. This behavior may be attributed to the presence of fewer number of charge carriers arising out of the very small concentration of salt. The VTF dependence of conductivity of samples shown in Figure $5 \mathrm{~A}$ indicates that the conductivity of the samples is coupled to the flow behavior of the polymer. The motion of ions in these samples appears to occur by a liquid like mechanism in which the movement of the ions through

Figure 5. Temperature dependence of conductivity of TPU 2 with various concentration of Li-perchlorate, $0.15 \mathrm{mmol} \mathrm{g}^{-1}$, $0.20 \mathrm{mmol} \mathrm{g}^{-1}$, and $\Delta 0.35 \mathrm{mmol} \mathrm{g}^{-1}$. (A) doped by solution blending, and (B) doped by absorption.

the polymer matrix is assisted by the large amplitude segmental motion of the polymer chains. As the samples presented in Figure 5A contains fully amorphous PPG in the soft segment and doping was done by more favorable solution blending method, it is more likely that the $\mathrm{Li}^{+}$ ions' movement will be more strongly assisted by the segmental motion of the PPO chains and hence the conductivity behavior is more VTF like.

As is evident from Figures 4, 5, and 6, the conductivity behavior of the TPU electrolytes with salt concentration is complex in nature. At each isotherm, the conductivity was found to increase with increase in salt concentration, except in one case (Figure 5A, curves 2 and 3). In Figure 5A, the conductivity of the sample containing $0.35 \mathrm{mmol}^{\circ} \mathrm{LiClO}_{4}$ per gm of TPU 2 is lower than that 

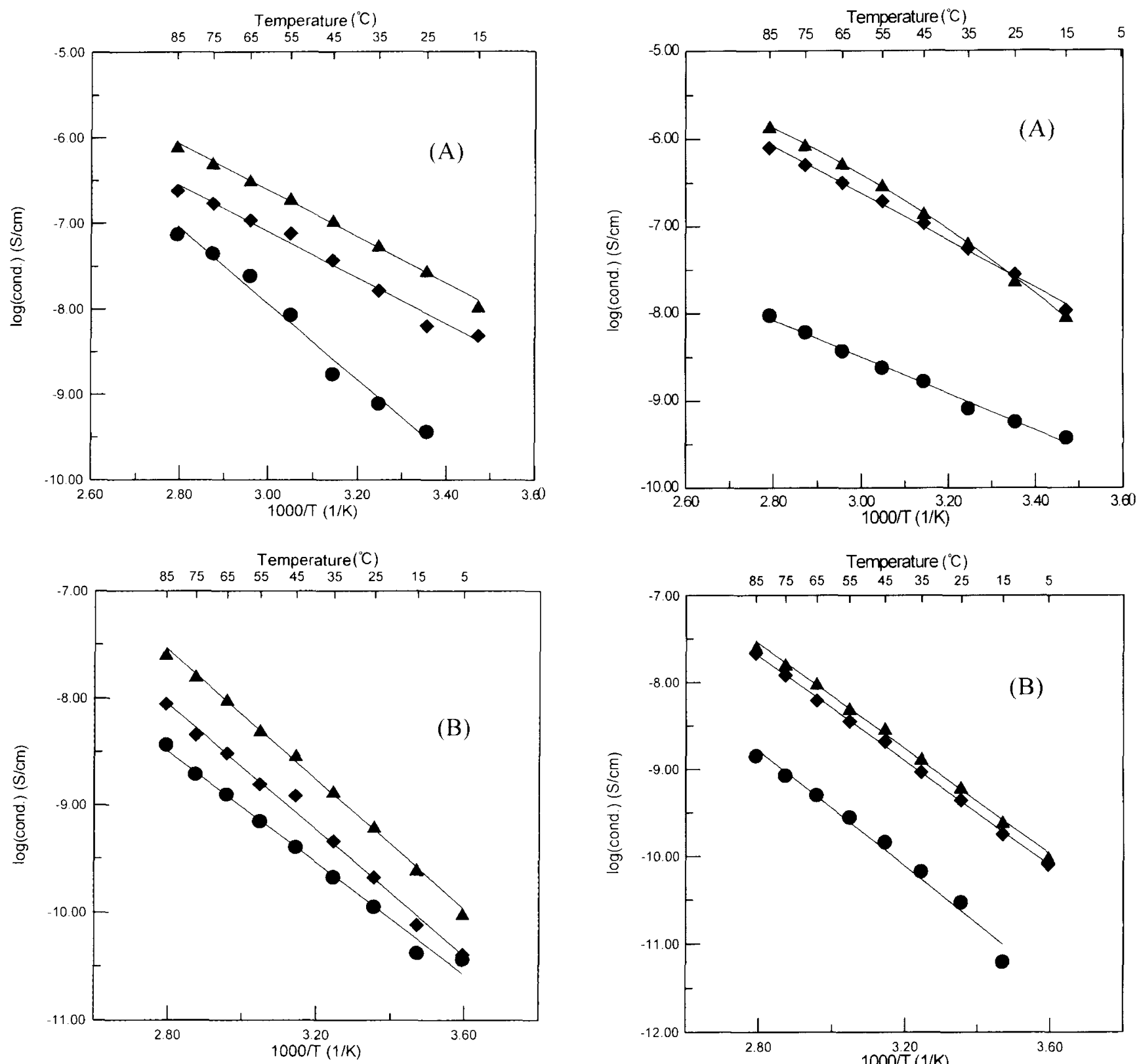

Figure 6. Temperature dependence of conductivity of TPU 3 with various concentration of $\mathrm{Li}$-perchlorate, $0.15 \mathrm{mmol} \mathrm{g}^{-1}$, $0.25 \mathrm{mmol} \mathrm{g}^{-1}$, and $\boldsymbol{\Delta} 0.35 \mathrm{mmol} \mathrm{g}^{-1}$. (A) doped by solution blending, and (B) doped by absorption.

of the sample containing $0.20 \mathrm{mmol}$ of $\mathrm{LiClO}_{4}$ per $\mathrm{gm}$ of TPU 2 at the lower temperature region, whereas the reverse phenomenon was observed at the higher temperature region. This phenomenon may be attributed to the formation of ion pairs, which increases with, increase in salt concentration. ${ }^{36}$ This leads to lesser number of free ions and also the mobility is limited, hence the conductivity is lower at lower temperature. At higher temperature, some of the ion pairs dissociate into free ions and hence the conductivity is increased at higher temperature.

The highest conductivity of TPU 2 may be due to the fully amorphous nature of the PPG soft segment, where the movement of the charge carriers is assisted by the large amplitude segmental motion of the PPG chains. Higher interaction of the $\mathrm{Li}^{+}$ions with PPG is also re-

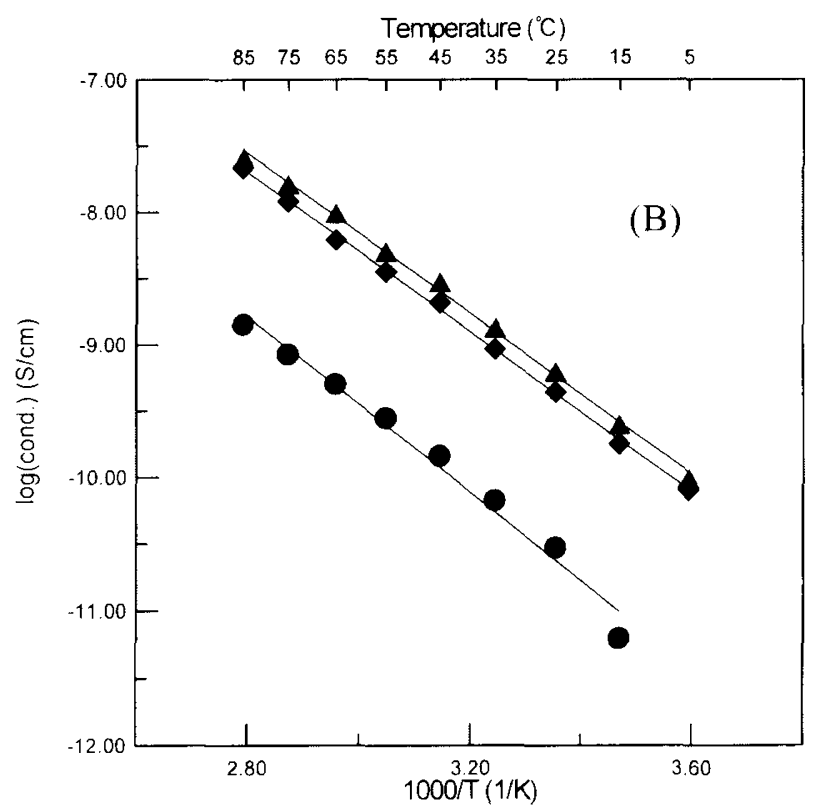

Figure 7. Comparison of temperature dependence of conductivity of TPU 1, TPU 2, and TPU 3 having $0.35 \mathrm{mmol} \mathrm{Li}$-perchlorate $\mathrm{g}^{-1}$. TPU (A) doped by solution blending and (B) doped by absorption, TPU $1, \diamond$ TPU 2 , and $\Delta$ TPU 3 .

flected from our FT-IR data, where both the H-bondedNH bands (peaks 3 and 4) were found to shift to lower frequency. No shifting was observed for peak 1 in case of TPU 2. This indicates that most of the $\mathrm{Li}^{+}$ions are associated to the soft segment. Although TPU 1 has lowest soft segment $T_{\mathrm{g}}$ (which is one of the requirement for increased mobility of the $\mathrm{Li}^{+}$ions and hence to higher conductivity), the semi crystalline nature of the soft segment (PTMG) of TPU 1 may be responsible for its low conductivity. The lower conductivity of TPU 1 is in line with our FT-IR data where almost no shifting of peak 4 was observed for TPU 1 with increase in salt concentration. This indicates weaker interaction of $\mathrm{Li}^{+}$ions with ether oxygens of TPU 1 soft segment in comparison to TPU 2 and TPU 3.

It is worthwhile to mention that conductivity of TPU 3 


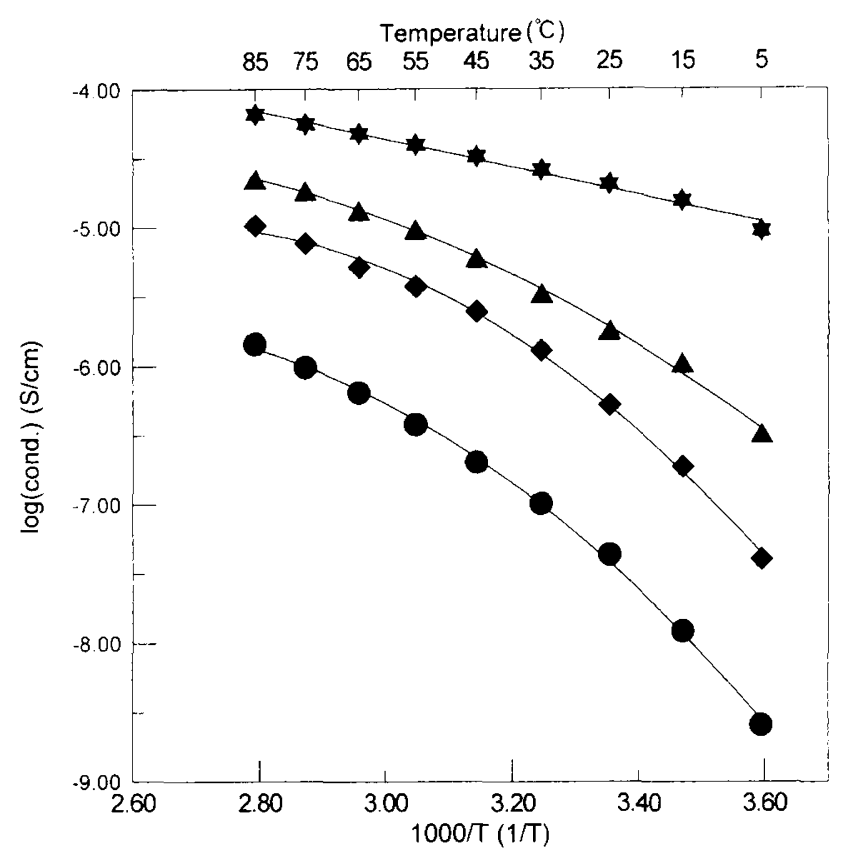

Figure 8. Temperature dependence of conductivity of TPU 3-S2 having different amount of added PC. $9.84 \%$, $19.67 \%$, $\Delta$ $30.62 \%$, and $\$ 39.81 \%$.

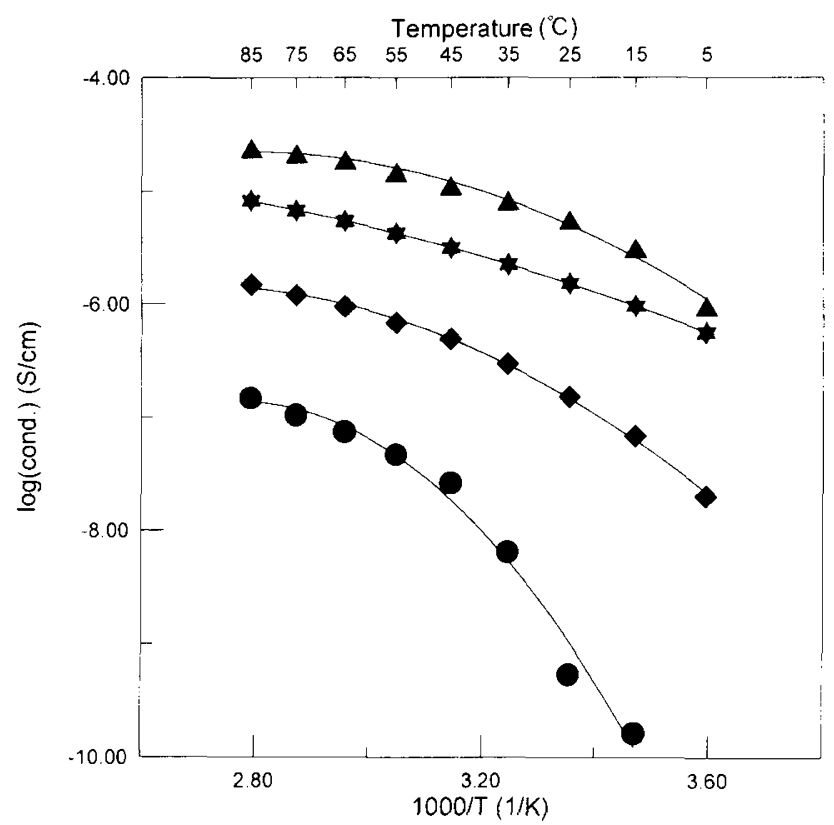

Figure 9. Temperature dependence of conductivity of TPU 3-A2 having different amount of added PC. $10.71 \%, \downarrow 19.34 \%$, $30.00 \%$, and $\boldsymbol{\Delta} 40.19 \%$.

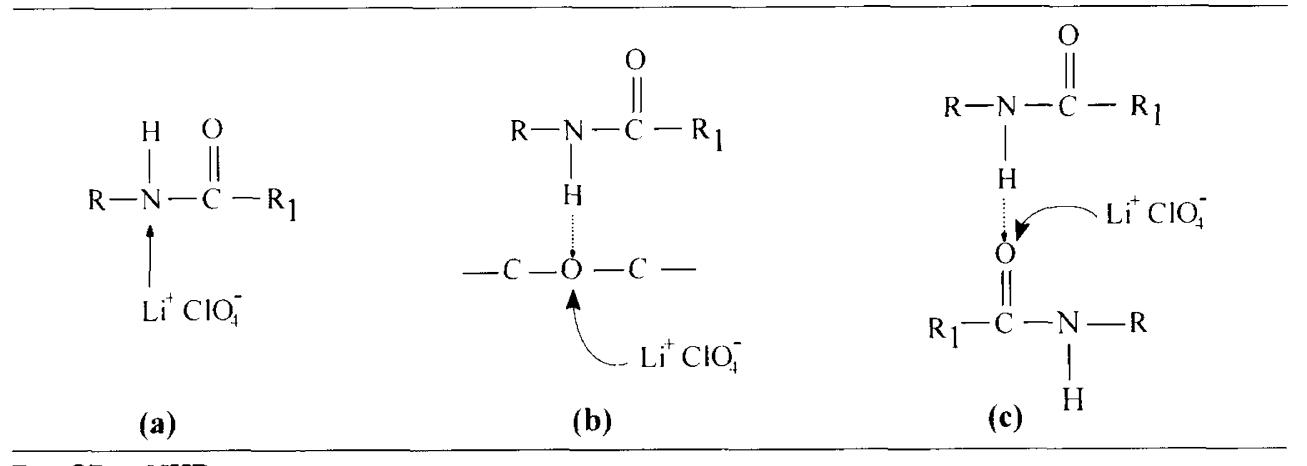

$\mathrm{R}_{1}=\mathrm{OR}$ or NHR

Scheme 2. Schemetic representation of coordination of $\mathrm{Li}^{+}$ions to different positions: (a) to the nitrogen atoms of free - $\mathrm{NH}$ groups, (b) to the hydrogen bonded ether oxygens, and (c) to the hydrogen bonded carbonyl oxygens.

is intermediate to TPU 1 and TPU 2 at each doping level and irrespective of the doping method (Figure 7). At lower doping level, the conductivity of TPU 3 is about an order of magnitude lower than TPU 2 throughout the temperature range, especially when doped by solution blending method. But at relatively higher doping level $\left(0.35 \mathrm{mmol} \mathrm{g}^{-1}\right)$, the conductivity of TPU 3 is more or less comparable to TPU 2. This result indicates that TPU 3 with $0.35 \mathrm{mmol}$ of $\mathrm{LiClO}_{4}$ per gm of polymer is as good an electrolyte as TPU 2. The reason for such increase in conductivity may be due to increased interaction of $\mathrm{Li}^{+}$ions with the soft segment. Our FT-IR data of TPU3 also indicate this behavior where very small shifting was observed at lower concentration of $\mathrm{Li}$ salt, but at higher concentration of salt the shifting is appreciable.

It is to be mentioned that for the GPEs, the temperature variation of ionic conductivity is VTF like, which is in contrast to the dry films where Arrhenius like behavior was observed. However, at high plasticizer concentration, transformation into Arrhenius like temperature dependence was observed. This behavior may be ex- plained in the following way. In GPEs, although the role of the matrix polymer is secondary (most of the ions are solvated and transported by the plasticizer), at lower concentration of plasticizer the matrix polymer plays a significant role in solvating and transporting the charge carriers. When the plasticizer concentration is low, some of the ions are residing in a polymer rich phase where the movement of the ions is associated with the segmental motion of the polymer chains. With higher amount of $\mathrm{PC}$, the role of the matrix polymer becomes negligible and the system becomes more liquid-like. In this situation, the mobility of the charge carriers become less coupled with the segmental motion of the polymer chains and hence the temperature dependence of ionic conductivity follows the Arrhenius equation.

\section{CONCLUSION}

A new segmented polyurethane based polymeric electrolyte has been made by using a 1:1 mixture of PPG and PTMG as soft segment and MDI extended with EDA as 
hard segment. DSC, FT-IR, and AC impedance results of this TPU doped with various concentration of $\mathrm{LiClO}_{4}$ were studied and compared with the parent TPU systems containing either PPG or PTMG as the soft segment. The newly made TPU containing PPG and PTMG in 1:1 molar ratio as the soft segment shows comparable conductivity $\left(\sim 10^{-6} \mathrm{~S} \mathrm{~cm}^{-1}\right.$ at $\left.85^{\circ} \mathrm{C}\right)$ to the TPU made using PPG only when studied as solid polymer electrolyte. About 2 orders of magnitude increase in conductivity was observed when the SPE was plasticized with $40 \%$ PC. The dimensional stability of this TPU is much better than that containing PPG, rather it is comparable to the TPU containing PTMG as the soft segment. Hence it can be concluded that this new TPU with both PPG and PTMG in the soft segment is a suitable matrix material for making polymer electrolytes where high conductivity and good dimensional stability is a prerequisite at high temperature.

Acknowledgment. The financial support of this work by National Science Council in Taiwan under NSC 882622-E006-008 and NSC 89-2214-E006-012 is gratefully acknowledged.

\section{REFERENCES}

1. T. M. Doscher, G. C. Myers, and D. C. Atkins Jr., J. Colloid. Sci., 6, 223 (1951).

2. A. A. Blumberg, S. S. Pollack, and C. A. Hoeve, J. Polym. Sci., 2, 2499 (1964)

3. J. Mocanin and E. F. Cuddihy, J. Polym. Sci., 14, 313 (1966).

4. R. D. Lundberg, F. Bailey, and R. W. Callard, J. Polym. Sci., 4, 1563 (1966).

5. R. Iwamoto, Y. Saito, H. Ishihara, and H. Tadokoro, J. Polym. Sci., 6, 1509 (1968).

6. A. E. Binks and A. Sharples, J. Polym. Sci., 6, 407 (1968).

7. M. Yokoyama, H. Ishihara, R. Iwamoto, and H. Tadokoro, Macromolecules, 2, 184 (1969).

8. B. E. Fenton, J. M. Parker, and P. V. Wright, Polymer, 14, 589 (1973).

9. P. V. Wright, Brit. Polym. J., 7, 319 (1975).

10. M. B. Armand, J. M. Chabgno, and M. J. Duclot, Second International Conference on Solid Electrolytes, St. Andrews, paper 6.5 (1978).

11. M. B. Armand, J. M. Chabgno, and M. J. Duclot, in "Fast Ion Transport in Solids", P. Vashishta, J. N. Mundy, and G. K. Shenoy, Ed., Elsevier North Holland, New York, 1979, p 131.
12. M. A. Ratner and D. F. Shriver, MRS Bulletin, 14, 39 (1989).

13. J. R. MacCullum and C. A. Vincent, Ed., "Polymer Electrolyte Reviews -I", Elsevier, London, 1987, p 1.

14. P. M. Blonsky, D. F. Shriver, P. Austin, and H. R. Allcock, J. Am. Chem. Soc., 106, 6854 (1984).

15. K. M. Abraham, in "Application of Electroactive Polymers", B. Scrosati, Ed., Chapman \& Hall, London, 1993, p 87.

16. S. L. Cooper and A. V. Tobolsky, J. Appl. Polym. Sci., 11, 1361 (1967).

17. V. W. Srichatrapimuk and S. L. Cooper, J. Macromol. Sci. Phys., B15, 267 (1978).

18. G. A. Senich and W. J. MacKnight, Macromolecules, 13, 106 (1980).

19. E. Santaniello, A. Manzocchi, and P. Sozzani, Tetrahedron Letts., 47, 4581 (1979).

20. M. M. Coleman, K. H. Lee, D. J. Skrovanek, and P. C. Painter, Macromolecules, 19, 2149 (1986).

21. M. M. Coleman, D. J. Skrovanek, J. Hu, and P. C. Painter, Macromolecules, 21, 59 (1988).

22. H. S. Lee, Y. K. Wang, and S. L. Hsu, Macromolecules, 20, 2089 (1987).

23. R. W. Seymour and S. L. Cooper, Macromolecules, 6, 48 (1973).

24. G. L. Wilkes, S. Bagrodia, W. Humphires, and R. Wildnauer, J. Polym. Letts., 13, 321 (1975).

25. G. L. Wilkes and R. Wildnauer, J. Appl. Phys., 46, 4148 (1975).

26. T. R. Hesketh, J. W. C. VanBogart, and S. L. Cooper, Polym. Eng. Sci., 20, 190 (1980).

27. L. M. Leung and J. T. Koberstein, Macromolecules, 19, 706 (1986).

28. J. T. Koberstein and A. F. Galambos, Macromolecules, 25, 5618 (1992).

29. A. W. McLennaghan and R. A. Pethrick, Eur. Polym. J., 24, 1063 (1988).

30. A. W. McLennaghan, A. Hooper, and R. A. Pethrick, Eur. Polym. J., 25, 1297 (1989).

31. M. Watanabe, S. Oohashi, K. Sanui, T. Kobayashi, and Z. Ohtaki, Macromolecules, 18, 1945 (1985).

32. M. Watanabe, K. Sanui, and N. Ogata, Macromolecules, 19, 815 (1986)

33. J. D. van Heumen and J. R. Stevens, Macromolecules, 28, 4268 (1995).

34. M. Seki and K. Sato, Makromol. Chem., 193, 2971 (1992).

35. T. C. Wen, M. S. Wu, and C. H. Yang, Macromolecules, 32, 2971 (1999).

36. S. Schantz, L. M. Torell, and J. R. Stevens, J. Chem. Phys., 94, 6862 (1991).

37. C. B. Wang and S. L. Cooper, Macromolecules, 16, 775 (1983). 